\title{
HMG-CoA reductase inhibitors (statins) increase endothelial progenitor cells via the PI 3-kinase/Akt pathway
}

\author{
Stefanie Dimmeler, ${ }^{1}$ Alexandra Aicher, ${ }^{1}$ Mariuca Vasa, ${ }^{1}$ Christiane Mildner-Rihm, ${ }^{1}$ \\ Klaudia Adler, ${ }^{1}$ Michaela Tiemann, ${ }^{2}$ Hartmut Rütten, ${ }^{2}$ Stephan Fichtlscherer, ${ }^{1}$ \\ Hans Martin, ${ }^{3}$ and Andreas M. Zeiher ${ }^{1}$
}

\author{
${ }^{1}$ Division of Molecular Cardiology, Department of Medicine IV, University of Frankfurt, Frankfurt, Germany \\ ${ }^{2}$ Aventis Pharmaceuticals, Frankfurt, Germany \\ ${ }^{3}$ Division of Hematology, Department of Medicine III, University of Frankfurt, Frankfurt, Germany \\ Address correspondence to: Stefanie Dimmeler, Molecular Cardiology, Department of Medicine IV, \\ University of Frankfurt, Theodor Stern-Kai 7, 60590 Frankfurt, Germany. \\ Phone: 49-69-6301-7440; Fax: 49-69-6301-7113; E-mail: Dimmeler@em.uni-frankfurt.de.
}

Received for publication April 27, 2001, and accepted in revised form June 11, 2001.

\begin{abstract}
HMG-CoA reductase inhibitors (statins) have been developed as lipid-lowering drugs and are well established to reduce morbidity and mortality from coronary artery disease. Here we demonstrate that statins potently augment endothelial progenitor cell differentiation in mononuclear cells and CD34-positive hematopoietic stem cells isolated from peripheral blood. Moreover, treatment of mice with statins increased $\mathrm{c}-\mathrm{kit}^{+} / \mathrm{Sca}-1^{+}-$positive hematopoietic stem cells in the bone marrow and further elevated the number of differentiated endothelial progenitor cells (EPCs). Statins induce EPC differentiation via the PI 3-kinase/Akt (PI3K/Akt) pathway as demonstrated by the inhibitory effect of pharmacological PI3K blockers or overexpression of a dominant negative Akt construct. Similarly, the potent angiogenic growth factor VEGF requires Akt to augment EPC numbers, suggesting an essential role for Akt in regulating hematopoietic progenitor cell differentiation. Given that statins are at least as potent as VEGF in increasing EPC differentiation, augmentation of circulating EPC might importantly contribute to the well-established beneficial effects of statins in patients with coronary artery disease.
\end{abstract}

J. Clin. Invest. 108:391-397 (2001). DOI:10.1172/JCI200113152.

\section{Introduction}

Stimulation of neovascularization is a therapeutic goal to rescue tissue from critical ischemia (1). Postnatal neovascularization was attributed mainly to angiogenesis, a process that is mediated by proliferation, migration, and remodeling of preexisting endothelial cells (2-4). However, recent studies provide increasing evidence that postnatal neovascularization does not rely exclusively on sprouting of preexisting vessels, but also involves bone marrow-derived circulating endothelial cells (5-7). A specific subset of leukocytes positive for CD34 was shown to home to sites of ischemia and express endothelial antigens $(5,8)$. Moreover, endothelial progenitor cells (EPCs) can be grown out of isolated $\mathrm{CD} 133^{+}$or $\mathrm{CD} 34^{+}$cells in vitro $(5,9,10)$ and make a significant contribution to adult blood vessel formation (8). Importantly, injection of isolated $\mathrm{CD} 34^{+}$cells or cultivated EPCs accelerates the restoration of blood flow in diabetic mice (11) and enhances neovascularization in vivo $(7,8,12,13)$. In addition, increased neovascularization induced by hematopoietic CD34-positive cells or angioblasts was shown recently to improve cardiac function $(14,15)$. Therefore, the stimulation of mobilization and/or differentiation of EPCs may provide a useful novel therapeutic strategy to improve postnatal angiogenesis. Proangiogenic growth factors such as VEGF (16) or GM-CSF therapy (17) augmented EPC levels. Moreover, VEGF 165 gene therapy increased the number of circulating differentiated EPCs in patients with limb ischemia (18). However, the mechanisms, which regulate EPC mobilization and differentiation, are not fully elucidated.

HMG-CoA reductase inhibitors (statins) have been developed as lipid-lowering drugs and are well established to reduce morbidity and mortality from coronary artery disease (19). Besides lipid-lowering, statins are capable of reducing vascular inflammation (20), decreasing platelet aggregation and thrombus deposition (21), and increasing endothelium-derived nitric oxide production (22). Statins have recently been shown to stimulate the growth of new blood vessels in ischemic limbs of normocholesterolemic rabbits (23). To gain insights into the mechanism by which statins improve neovascularization, we investigated the effects of statins on circulating bone marrow-derived endothelial cells and elucidated the underlying signal transduction pathways.

The present study demonstrates that statins augment EPC numbers in vitro and in mice. The increase in EPC levels induced by both statins and VEGF was mediated via the activation of the serine/threonine kinase Akt, 
which is known to play an important role in endothelial biology and angiogenesis $(23,24)$.

\section{Methods}

Cell culture. Total mononuclear cells (MNCs) were isolated from blood of healthy human volunteers by density gradient centrifugation with Biocoll separating solution (density 1.077 (Biochrom AG, Berlin, Germany). MNCs $\left(4 \times 10^{6}\right)$ were plated in $0.5 \mathrm{ml}$ endothelial basal medium (EBM) (CellSystems, St. Katharinen, Germany), with supplements $(1 \mu \mathrm{g} / \mathrm{ml}$ hydrocortisone, $3 \mu \mathrm{g} / \mathrm{ml}$ bovine brain extract, $30 \mu \mathrm{g} / \mathrm{ml}$ gentamicin, 50 $\mu \mathrm{g} / \mathrm{ml}$ amphotericin B, $10 \mu \mathrm{g} / \mathrm{ml} \mathrm{EGF}$, and 20\% FCS) on fibronectin/gelatin-coated 24-well plates. After 3 days of culture, MNCs were stimulated with human recombinant VEGF (Biomol Feinchemikalien GmbH, Hamburg, Germany) or activated simvastatin, mevastatin (Calbiochem-Novabiochem GmbH, Bad Soden, Germany) or atorvastatin for the respective time points. Atorvastatin was kindly donated by Goedecke/Parke-Davis (Freiburg, Germany). Medium was then changed, and adherent cells were washed

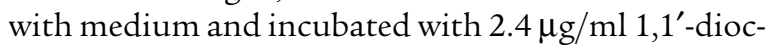
tadecyl-3,3,3',3'-tetramethylindocarbocyanine-labeled acetylated LDL (DiLDL; Harbor Bio-Products, Norwood, Massachusetts, USA) for 1 hour. Cells were fixed in $2 \%$ paraformaldehyde and counterstained with FITC-labeled lectin from Ulex europaeus (Sigma RBI, Taufkirchen, Germany). Two to three independent investigators evaluated the number of EPCs per well by counting three randomly selected high-power fields.

MNCs were transfected after 3 days of culture with pcDNA3.1.-GFP, pcDNA3.1 (empty vector), or pcDNA3.1.-myc-his dominant negative Akt (25) using Lipofectamine PLUS (Life Technologies, Karlsruhe, Germany). Then, $0.5 \mu \mathrm{g}$ plasmid, $4 \mu \mathrm{l}$ PLUS, and 150 $\mu \mathrm{EBM}$ medium were incubated for 15 minutes followed by a mixture of $150 \mu \mathrm{l}$ EBM medium and $1 \mu \mathrm{l}$ Lipofectamine. Cells were washed once with EBM medium and incubated with the mixture and $1 \mathrm{ml}$ EBM medium for 3 hours. Then, $1 \mathrm{ml}$ EBM medium was added, and cells were incubated for a further 3 hours before medium was changed.

Isolation of $\mathrm{CD} 34^{+}$cells. Human $\mathrm{CD} 34^{+}$cells were isolated from leukapheresis products from chemotherapy and G-CSF-mobilized patients with non-Hodgkin lymphoma after informed consent. After incubation with immunomagnetic CD34-microbeads (Miltenyi Biotec, Bergisch-Gladbach, Germany) for 30 minutes at $4^{\circ} \mathrm{C}$, cells were washed in PBS plus $0.5 \%$ BSA plus 2 mM EDTA, filtered through a $40-\mu \mathrm{m}$ cell strainer, and run over a magnetic cell separation device (Auto-Macs; Miltenyi Biotec) for positive selection of $\mathrm{CD} 34^{+}$cells. Isolated CD $34^{+}$cells were cultured in fibronectin-coated 24-well plates (Greiner Labortechnik GmbH, Frickenhausen, Germany) at a density of 300,000 cells $/ 0.5$ $\mathrm{ml}$ EBM medium for 48 hours.

Animal experiments. Age-matched C57BL/6 male mice (The Jackson Laboratory, Bar Harbor, Maine, USA) were fed with a daily oral dose of $20 \mathrm{mg} / \mathrm{kg}$ simvastatin for 3 weeks ( $n=10$ animals). Control mice ( $n=15$ animals) were kept without simvastatin. Both groups of mice were killed to obtain specimens of spleen and bone marrow. Spleens were mechanically minced using syringe plungers and laid over Ficoll to isolate MNCs (splenocytes). Splenocytes $\left(8 \times 10^{6}\right)$ were seeded into fibronectin-coated 24-well plates in $0.5 \mathrm{ml} \mathrm{EBM}$ medium. After 48 hours, medium was removed and adherent cells were stained for Dil-Ac-LDL/lectin as described above. Bone marrow was harvested by flushing femurs and tibias with RPMI-1640 medium containing 10\% FCS and further analysed by FACS; c-kit/sca-1 double staining was only performed in a subset of animals.

FACS analysis. For FACS analysis of human EPCs, the adherent cells were gently scraped off using cell scrapers (Greiner Labortechnik $\mathrm{GmbH}$ ) and washed in PBS. Cells were incubated in PBS plus 1\% BSA plus 1\% mouse serum in the presence of the following $\mathrm{mAb}$ 's. Staining of mouse anti-human VE-cadherin (Santa Cruz Biotechnology Inc., Heidelberg, Germany) and KDR (Dianova, Hamburg, Germany) was visualized using RPE-conjugated goat anti-mouse $\mathrm{F}\left(\mathrm{ab}^{\prime}\right) 2$ (DAKO Corp., Hamburg, Germany). The anti-CD31 Ab was directly linked to FITC. Rabbit anti-human vWF Ab (Calbiochem-Novabiochem $\mathrm{GmbH}$ ) was used in combination with FITC-linked anti-rabbit secondary Ab. The secondary step reagent without primary $\mathrm{Ab}$ was used as a negative staining control.

FACS staining of murine cells from bone marrow was done in PBS plus $1 \%$ BSA plus $1 \%$ rat/rabbit serum with the following directly conjugated $\mathrm{mAb}$ 's: rat-anti mouse c-kit (CD117)-FITC and sca-1-phycoerythrin (sca-1-PE) (BD Biosciences, Heidelberg, Germany), as well as their corresponding isotype-matched FITC or PE-conjugated rat immunoglobulins. Single- and two-color flow cytometric analyses were performed using a FACScan flow cytometer and Cell Quest software (BD Biosciences). Each analysis included at least 3,000 events.

\section{Results}

Statins increase EPCs in vitro. Incubation of isolated human MNCs with atorvastatin dose and time dependently increased the number of differentiated, adherent EPCs from $2.1 \% \pm 0.16 \%$ to $5 \% \pm 0.35 \%$ of the total number of MNCs in vitro (Figure 1, a and b). Similarly, the HMG-CoA reductase inhibitors mevastatin $(1 \mu \mathrm{M})$ and simvastatin $(1 \mu \mathrm{M})$ also augmented the number of EPCs up to $288 \% \pm 17 \%$ and $241 \% \pm 102 \%$, respectively (Figure 1c). EPCs were characterized as adherent cells double positive for DiLDL-uptake and lectin binding $(5,18)$ (Figure 1c). The endothelial phenotype of the EPCs was additionally confirmed by documenting the expression of well-established cell surface markers like vWF, VEGF-receptor 2 (KDR-receptor), VE-cadherin, and CD31 by fluorescence-activated cell sorting (FACS) and immunostaining (Figure $1 \mathrm{~d}$ and data not shown). The expression pattern of these endothelial cell mark- 
a

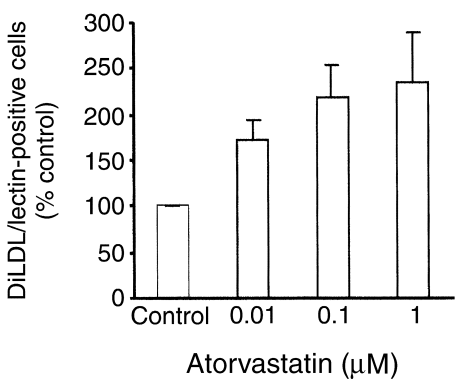

C
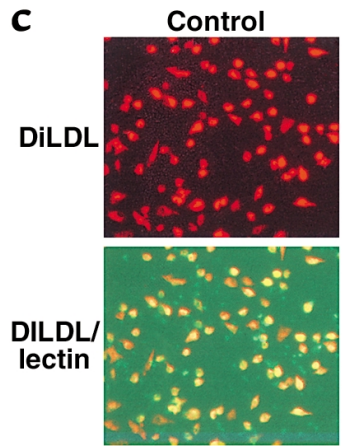

d

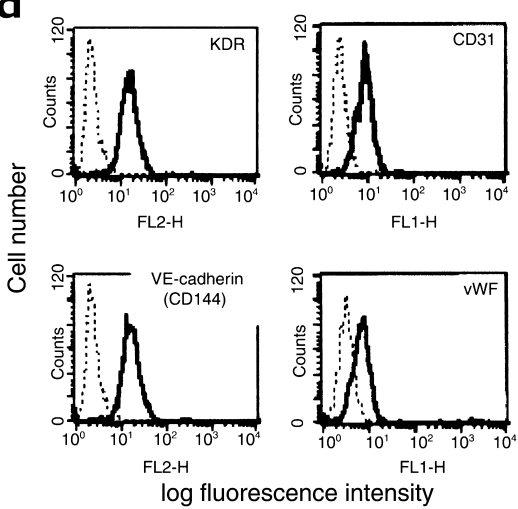

b
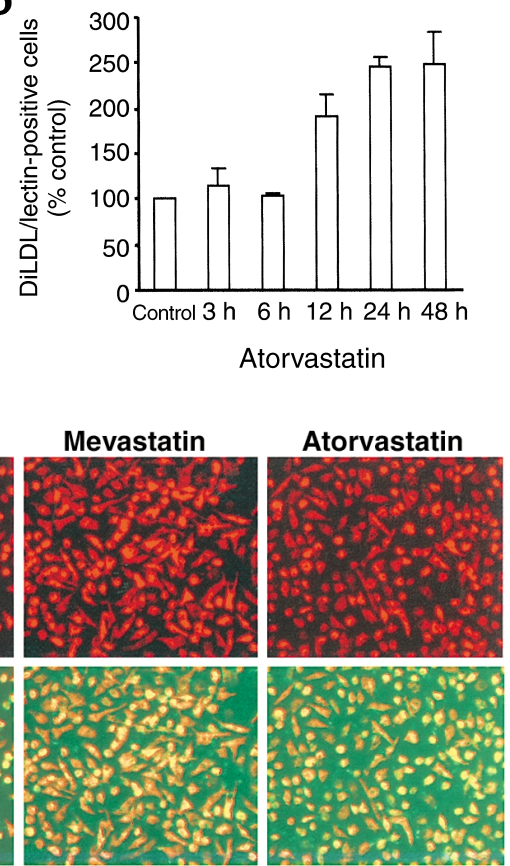

e

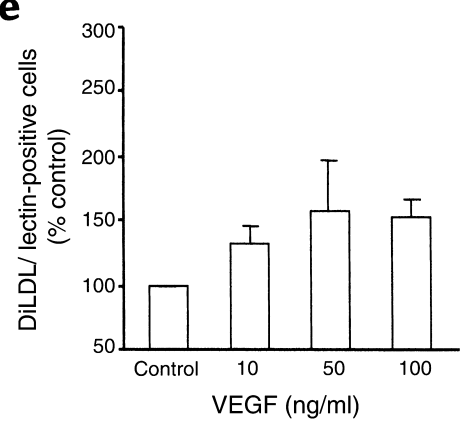

\section{Figure 1}

Statins increase the number of adherent EPCs. ( $\mathbf{a}$ and $\mathbf{b}$ ) MNCs were incubated with atorvastatin as indicated, and adherent DiLDL/lectinpositive cells were counted (13). Basal control values represent $388 \pm 146$ cells $/ \mathrm{mm}^{2}$. The solvent DMSO had no effect on EPC differentiation. Data are mean \pm SEM, $n=4-6$. (c) MNCs were incubated with atorvastatin $(1 \mu \mathrm{M})$, simvastatin $(1 \mu \mathrm{M})$, or mevastatin $(1 \mu \mathrm{M})$ for 24 hours, and adherent cells' DiLDL uptake (red) and lectin binding (green) were assessed. Double positive cells appear yellow in the overlay. Representative images are shown from at least three experiments. (d) MNCs were incubated for 4 days. Adherent cells were analysed for expression of KDR, CD31, VE-cadherin, and VWF by FACS. Dotted lines represent isotype controls. Similar expression patterns were observed after stimulation of MNCs with atorvastatin ( $1 \mu \mathrm{M}, 24$ hours) (data not shown). Representative images from $n=4$ experiments are shown. (e) MNCs were incubated with VEGF for 24 hours and adherent DiLDL/lectin-positive cells were counted. Data are mean \pm SEM, $n=3-6$.

ers on EPCs was found to be comparable to that observed in human umbilical venous endothelial cells (HUVECs) (data not shown). In addition, the expression and activity of the endothelial nitric oxide synthase in EPCs was demonstrated by immunostaining, Western blot analysis, and DAF-2 staining, respectively (data not shown).

Because the angiogenic growth factor VEGF is known to augment the number of EPCs $(16,18)$, we compared the statin effect with VEGF. As illustrated in Figure 1e, human recombinant VEGF also stimulated the differentiation of EPCs with a maximal effect at $100 \mathrm{ng} / \mathrm{ml}$.
The effect of statins appears to be at least equipotent compared, with VEGF.

To exclude the possibility that the effect of VEGF and statins is due to increased adherence of adult circulating endothelial cells, we isolated $\mathrm{CD} 34^{+}$hematopoietic stem cells (HSCs), which presumably represent the EPC precursor cells in humans $(5,12,26)$. After separation of CD34-positive cells by immunomagnetic CD34 microbeads from mobilized peripheral blood, the purity of the population was $93.8 \%$ (range $82.4-99 \%$ ) as analysed by FACS (Figure 2a). Addition of VEGF or atorvastatin to the isolated $\mathrm{CD} 34^{+}$hematopoietic pro- 
a

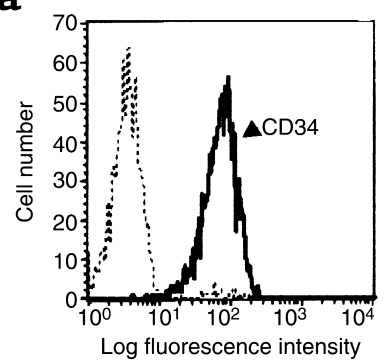

b

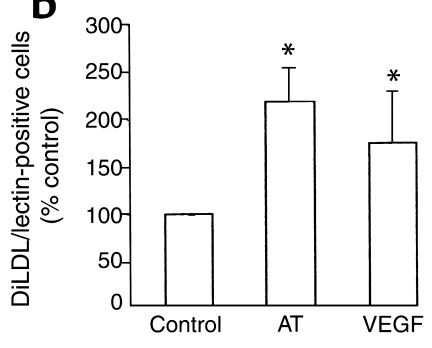

Figure 2

Statins and VEGF induce differentiation of $\mathrm{CD} 34^{+}$cells. (a) $\mathrm{CD} 34^{+}$cells were isolated and purity of $\mathrm{CD} 34^{+}$cell fraction was analysed by FACS. Isotype control is represented by a dotted line and CD34 by a solid line. (b) CD34 $4^{+}$cells were incubated with atorvastatin (AT; $1 \mu \mathrm{M})$ or VEGF $(100 \mathrm{ng} / \mathrm{ml})$ for 24 hours, and adherent DiLDL/lectin-positive cells were counted. Data are mean \pm SEM, $n=4-7,{ }^{*} P<0.05$ versus control. genitor cells significantly increased the number of differentiated EPCs (Figure 2b). Consistent with the effect obtained in MNCs, atorvastatin was at least as effective as VEGF (Figure 2b). Taken together, these data demonstrate that statins and VEGF induce differentiation of hematopoietic progenitor cells into EPCs.

Statins increase EPC levels in vivo. To test the in vivo relevance of our findings, mice were fed with simvastatin $(20 \mathrm{mg} / \mathrm{kg}$ daily) for 3 weeks, and EPC numbers were determined. As shown in Figure 3a, statin treatment led to a more than twofold increase in DiLDL/lectin-positive cells, thus extending the in vitro data. To gain further insights into the effect of statins, the number of multipotent bone marrow HSCs, which are characterized by expression of c-kit (CD117) and sca-1 $(26,27)$, was analysed. The number of c-kithigh cells was profoundly increased in bone marrow of simvastatin-fed mice (Figure 3b). Moreover, c-kit/sca-1 double positive bone marrow cells were increased from $0.78 \% \pm 0.3 \%$ in controls to $8.2 \% \pm 2.6 \%$ in simvastatin-treated mice $(P \leq 0.05)$. In contrast, the overall number of sca- $1^{\text {high }}$ cells did not significantly change $(37 \% \pm 6 \%$ in control versus $45 \% \pm 14 \%$ in simvastatin-fed mice). These data suggest that statins specifically augment multipotent c-kit/sca-1 HSCs in the bone marrow and concomitantly increase the number of differentiated EPCs in the periphery in vivo.

The effects of VEGF and statins on EPCs is mediated via the PI 3-kinase/Akt pathway. Next, we investigated the molecular mechanisms underlying the effects of statins on EPC differentiation. Since statins and VEGF have both been shown to stimulate the PI 3-kinase/Akt (PI3K/Akt) pathway $(23,28)$, we used the pharmacological PI3K-inhibitors Ly294002 and wortmannin to assess whether the PI3K-pathway is involved. Inhibi- tion of the PI3K-pathway abolished statin- and VEGFstimulated increase of EPCs (Figure 4, a and b). In contrast, inhibition of another VEGF-stimulated kinase cascade, namely the MAP-kinases ERK-1 and -2, by PD98059 did not significantly alter the differentiation of EPCs (Figure 4, a and b), suggesting that a PI3K rather than a MAP-kinase pathway mediates the effects of statins and VEGF.

To demonstrate that Akt is indeed an essential downstream signaling event, Akt phosphorylation indicative for Akt activity was detected by Western blot analysis. Statins and VEGF potently stimulated Akt phosphorylation in isolated MNCs (Figure 4c and data not shown). The causal contribution of Akt for statin- and VEGF-induced EPC differentiation was finally demonstrated by transient transfection of the MNCs with a dominant negative Akt construct. The transfection efficiency was about $50 \%$, as determined by GFP-fluorescent cells (Figure 4d). Overexpression of the dominant negative Akt inhibited the increase of EPCs induced by statins or VEGF (Figure 4e), clearly demonstrating that Akt is causally involved in EPC differentiation.

Finally, we attempted to characterize the molecular signal transduction pathway of statins to modulate EPC differentiation. The inhibition of HMG-CoA reductase, which mediates the cholesterol-lowering effect of statins, also reduces the formation of the metabolic products geranylgeranylpyrophosphat (GGPP) (22). Blockade of geranylgeranylation, in turn, abolishes Rho-kinase activation and increases eNOS mRNA stability (Figure 5a) $(22,29)$. Therefore, we determined the effect of mevalonate, the end product of the HMG-CoA reductase, and the further downstream intermediate GGPP. Mevalonate reversed the inhibitory effect of statins, whereas GGPP led only to
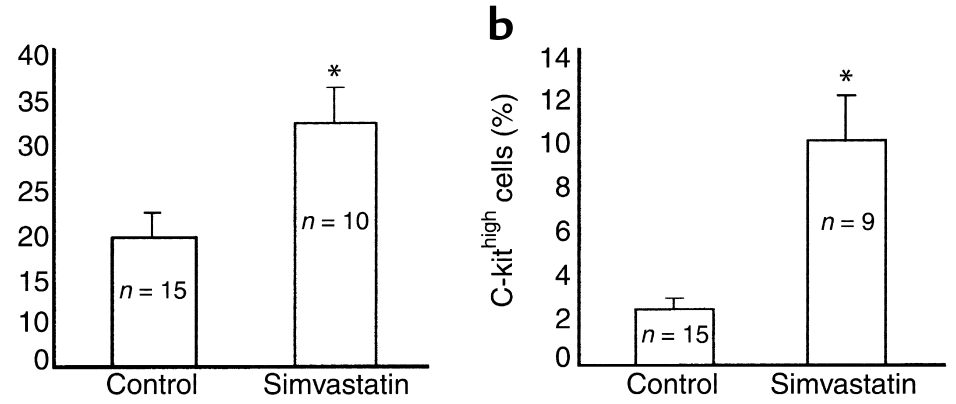

\section{Figure 3}

Statins increase EPCs and HSCs in mice. (a) Mice were fed with simvastatin for 3 weeks. Splenocytes were isolated and incubated for 4 days. Adherent DiLDL/lectin-positive cells were counted. Data are mean $\pm \mathrm{SEM},{ }^{*} P<0.05$ versus control. (b) Bone marrow cells were isolated and the number of c-kithigh cells was measured by FACS. Data are mean \pm SEM, ${ }^{*} P=0.006$ versus control (Mann-Whitney test). 

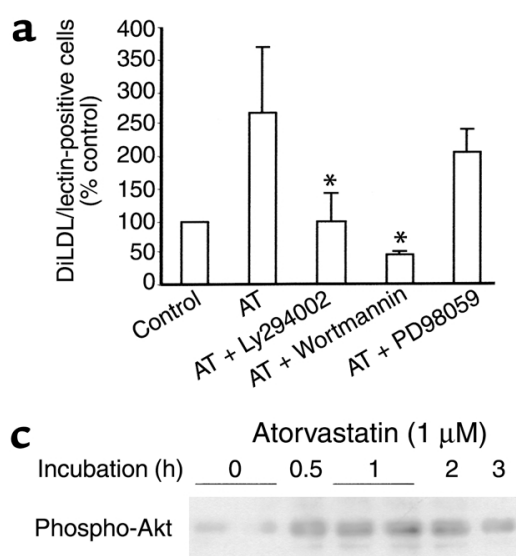

Akt

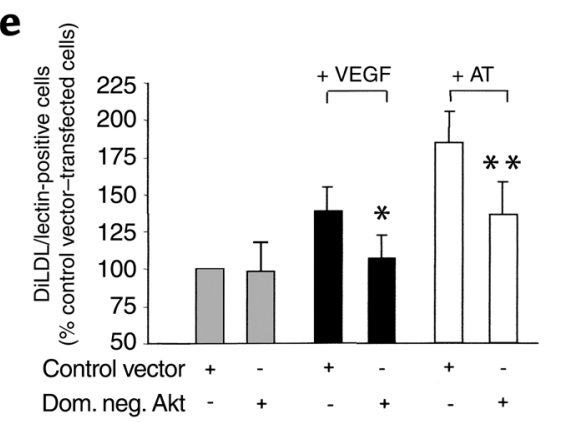

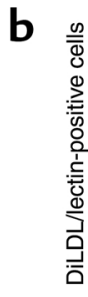

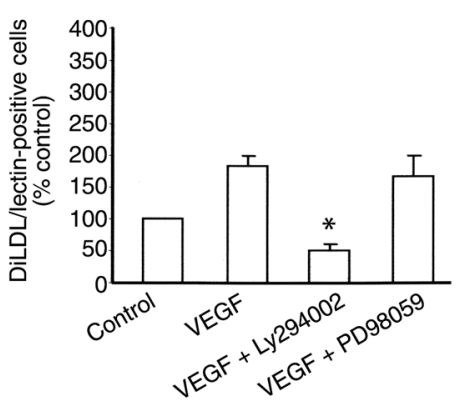

d

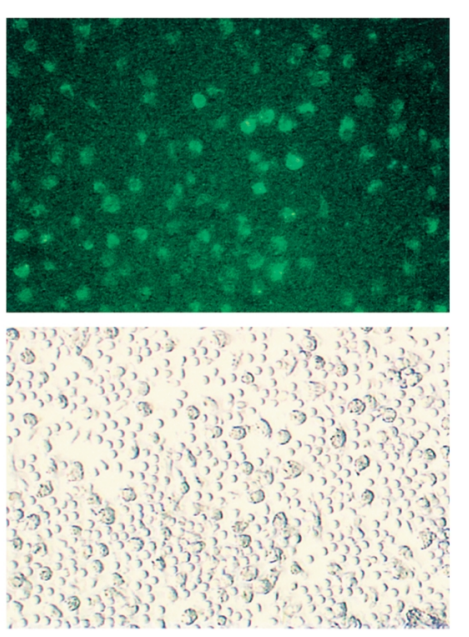

Figure 4

Statin- and VEGF-induced EPC differentiation is mediated by the PI3K/Akt pathway. ( $\mathbf{a}$ and $\mathbf{b}$ ) MNCs were incubated with atorvastatin $(1 \mu \mathrm{M})$ or VEGF $(100 \mathrm{ng} / \mathrm{ml})$ and the respective inhibitors (Ly294002, $10 \mu \mathrm{M}$; wortmannin, 10 nM; PD98059, $10 \mu \mathrm{M})$ for 24 hours. Adherent DiLDL/lectin-positive cells were counted. Neither the substances alone nor the solvents had any toxic effect (data not shown). Data are mean \pm SEM, $n=3-6,{ }^{*} P<0.05$ versus VEGF or AT. (c) Western blot against phosphorylated Akt (Ser 473) of MNCs incubated with atorvastatin for the respective time points. Equal loading was confirmed by reprobe of the membranes with total Akt. Protein isolation and Western blot analysis was performed as outlined previously (25). A representative experiment is shown $(n=3)$. ( $\mathbf{d}$ and $\mathbf{e})$ MNCs were transfected with pcDNA3.1.-GFP as control vector or pcDNA3.1. dominant negative (dom. neg.) Akt and were incubated with atorvastatin $(1 \mu \mathrm{M})$ or recombinant hVEGF $(100 \mathrm{ng} / \mathrm{ml})$ for 24 hours. Adherent DiLDL/lectin-positive cells were counted. Atorvastatin was similarly effective in empty vector-transfected cells compared with GFP. Data are expressed as mean \pm SEM, $n=4-6,{ }^{*} P<0.05$ versus control vector plus VEGF, ${ }^{*} P<0.05$ versus control vector plus atorvastatin. a minor decrease in atorvastatin-induced EPC differentiation (Figure $5 \mathrm{~b}$ ), suggesting that the downstream signaling of GGPP is less important to mediate the statin effects. Since statins exert an inhibitory effect on the Rho kinase, we also determined whether blocking of Rho kinase by the pharmacological inhibitor HA 1077 would increase EPC numbers. However, HA 1077 did not augment EPC numbers (Figure $5 \mathrm{~b}$ ). In accordance with these findings, the eNOS inhibitor $\mathrm{N}^{\mathrm{G}_{-}}$ mono-methyl-L-arginine did not inhibit statininduced increases in EPC numbers (Figure $5 b$ ), thus excluding a potential role of the Rho kinase/endothelial NO synthesis pathway.

\section{Discussion}

The data of the present study establish a novel mechanism of action of statins, namely the increase in EPC numbers. Although the proportional contribution of angiogenesis and vasculogenesis to neovascularization of adult tissue remains to be determined, it is well established that EPCs participate in repair following ischemic injury. Thus, increasing the number of circulating EPCs by transplantation of hematopoietic stem cells or by injection of in vitro-differentiated EPCs has been shown to improve neovascularization of ischemic hindlimb $(12,13)$, accelerate blood flow in diabetic mice (11), and improve cardiac function (14). Therefore, augmentation of circulating EPCs by statin ther- a

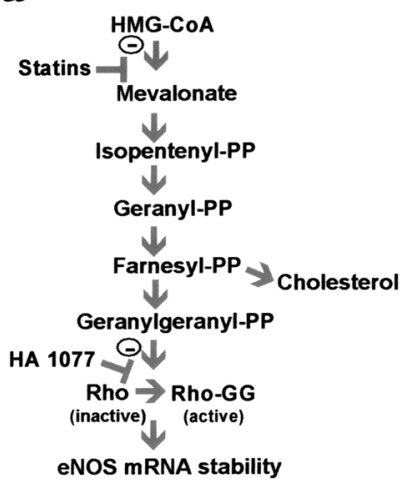

b

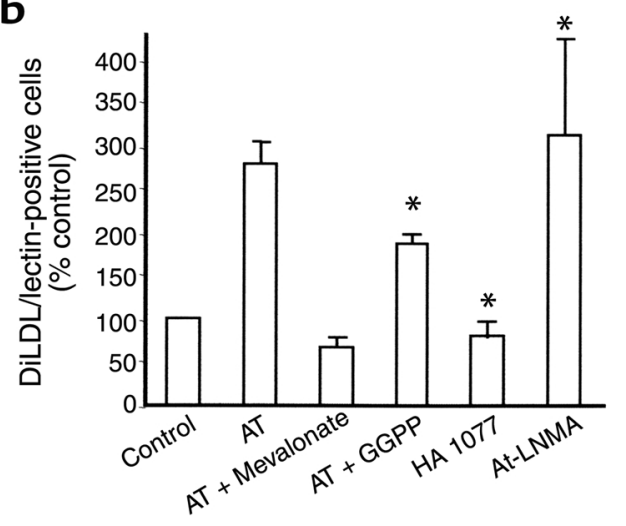

Figure 5

Characterization of the statin effect. (a) Schematic illustration of the statin effects on intracellular pathways. (b) MNCs were incubated with atorvastatin (AT; $1 \mu \mathrm{M})$ for 24 hours with or without mevalonate $(100 \mu \mathrm{M})$, GGPP $(5 \mu \mathrm{M})$, the Rhokinase inhibitor $\mathrm{HA} 1077(10 \mu \mathrm{M})$, or $\mathrm{NG}^{\mathrm{G}}$-monomethyl-L-arginin (LNMA, 1mM). DiLDL/lectinpositive cells were counted. Data are expressed as mean \pm SEM, $P<0.05$ versus AT; $n=3-7$. 
apy may significantly contribute to the stimulation of neovascularization after tissue ischemia. Recently, in fact, restatin therapy has not only been shown to rapidly enhance coronary blood flow in patients with stable coronary artery disease (30), but also to reduce myocardial ischemia in patients with acute coronary syndromes within a few weeks of treatment (31).

The increase in EPC number by statins is at least equipotent compared with the prototypic angiogenic cytokine VEGF, which is known to promote EPC mobilization and differentiation in vitro and after gene transfer in vivo $(5,16,18)$. One may speculate that statins induce the expression of angiogenic growth factors such as VEGF and thereby influence EPC numbers. However, atorvastatin did not affect expression of various angiogenic factors or their receptors in isolated EPCs, as demonstrated by mRNase protection assays (data not shown). Thus, it is unlikely that the effects of statins are mediated via upregulation of angiogenic growth factors. In contrast, the present data suggest that statins similar to VEGF - can directly induce differentiation of monocytes or CD34-positive hematopoietic precursor cells into EPCs. In vivo, statins further augment the levels of bone marrow hematopoietic precursor cells. Thus, statins may influence not only the differentiation process, but may also modulate mobilization of hematopoietic progenitor cells as shown for VEGF.

VEGF and statins appear to share a common $\mathrm{PI} 3 \mathrm{~K} /$ Akt signaling pathway to regulate EPC differentiation levels. The protein kinase Akt plays a central role in mature endothelial cells. Activation of Akt promotes endothelial cell survival by inhibiting apoptosis $(28,32)$, stimulates endothelial NO synthesis $(25,33)$, and mediates VEGF-induced endothelial cell migration $(34,35)$. The present study not only extends these findings by demonstrating that Akt regulates differentiation of EPCs, but also identified Akt as a target for statins to modify EPC kinetics, which might importantly contribute to the well-established beneficial effects of statin therapy in patients with myocardial ischemia due to obstructive coronary artery disease.

The question remains how statins can activate the PI3K. In accordance with the data of Kureishi and coworkers (23), we demonstrated that statin-induced activation of Akt in endothelial cells was due to inhibition of mevalonate formation, but largely independent of the downstream product GGPP. Furthermore, inhibition of NO-synthesis did not prevent statin-induced increases in EPC numbers in vitro. This is consistent with the finding that inhibition of the Rho kinase, which mediates the eNOS mRNA stabilizing effect of statins (22) and therefore should mimic the effect of statins on eNOS, did not affect EPC numbers.

In conclusion, the results of the present study demonstrate that statins can induce the differentiation of endothelial progenitor cells and upregulate EPC numbers in vitro and in vivo. In this way, the PI3K/Akt pathway appears to play a major role for statin- and VEGF-induced increases in EPC differentiation. The augmentation of EPC numbers by pharmacological modulation of the signaling pathways, which regulate EPC differentiation, may be a novel strategy to improve neovascularization after ischemia and, thereby, provide a therapeutic concept to improve EPC numbers in patients with coronary artery disease.

\section{Acknowledgments}

We would like to thank Marga Müller-Adorgan and Meike Stahmer for technical help. This study was supported by the Deutsche Forschungsgemeinschaft (SFB 335, B6) and by a cooperation with the Max-Planck Institute for Physiological and Clinical Research, Department of Experimental Cardiology (Bad Nauheim, Germany). Alexandra Aicher was supported by the KGF e.V. Frankfurt/Main (Bone Marrow Transplantation/Gene Therapy Association).

1. Isner, J.M., and Asahara, T. 1999. Angiogenesis and vasculogenesis as therapeutic strategies for postnatal neovascularization. J. Clin. Invest. 103:1231-1236.

2. Folkman, J. 1995. Angiogenesis in cancer, vascular, rheumatoid and other disease. Nat. Med. 1:27-31.

3. Risau, W. 1997. Mechanisms of angiogenesis. Nature. 386:671-674.

4. Carmeliet, P., and Jain, R.K. 2000. Angiogenesis in cancer and other diseases. Nature. 407:249-257.

5. Asahara, T., et al. 1997. Isolation of putative progenitor endothelial cells for angiogenesis. Science. 275:964-967.

6. Shi, Q., et al. 1998. Evidence for circulating bone marrow-derived endothelial cells. Blood. 92:362-367.

7. Asahara, T., et al. 1999. Bone marrow origin of endothelial progenitor cells responsible for postnatal vasculogenesis in physiological and pathological neovascularization. Circ. Res. 85:221-228.

8. Crosby, J.R., et al. 2000. Endothelial cells of hematopoietic origin make a significant contribution to adult blood vessel formation. Circ. Res. 87:728-730.

9. Gehling, U.M., et al. 2000. In vitro differentiation of endothelial cells from AC133-positive progenitor cells. Blood. 95:3106-3112.

10. Bhattacharya, V., et al. 2000. Enhanced endothelialization and microvessel formation in polyester grafts seeded with CD34(+) bone marrow cells. Blood. 95:581-585.

11. Schatteman, G.C., Hanlon, H.D., Jiao, C., Dodds, S.G., and Christy, B.A. 2000. Blood-derived angioblasts accelerate blood-flow restoration in diabetic mice. J. Clin. Invest. 106:571-578.

12. Murohara, T., et al. 2000. Transplanted cord blood-derived endothelial precursor cells augment postnatal neovascularization. J. Clin. Invest. 105:1527-1536.

13. Kalka, C., et al. 2000. Transplantation of ex vivo expanded endothelial progenitor cells for therapeutic neovascularization. Proc. Natl. Acad. Sci. USA. 97:3422-3427.

14. Kocher, A.A., et al. 2001. Neovascularization of ischemic myocardium by human bone-marrow-derived angioblasts prevents cardiomyocyte apoptosis, reduces remodeling and improves cardiac function. Nat. Med. 7:430-436

15. Kawamoto, A., et al. 2001. Therapeutic potential of ex vivo expanded endothelial progenitor cells for myocardial ischemia. Circulation. 103:634-637.

16. Asahara, T., et al. 1999. VEGF contributes to postnatal neovascularization by mobilizing bone marrow-derived endothelial progenitor cells. EMBOJ. 18:3964-3972.

17. Takahashi, T., et al. 1999. Ischemia- and cytokine-induced mobilization of bone marrow-derived endothelial progenitor cells for neovascularization. Nat. Med. 5:434-438.

18. Kalka, C., et al. 2000. Vascular endothelial growth factor(165) gene transfer augments circulating endothelial progenitor cells in human subjects. Circ. Res. 86:1198-202.

19. Maron, D.J., Fazio, S., and Linton, M.F. 2000. Current perspectives on statins. Circulation. 101:207-213.

20. Bustos, C., et al. 1998. HMG-CoA reductase inhibition by atorvastatin reduces neointimal inflammation in a rabbit model of atherosclerosis. J. Am. Coll. Cardiol. 32:2057-2064.

21. Lacoste, L., et al. 1995. Hyperlipidemia and coronary disease. Correction of the increased thrombogenic potential with cholesterol reduction. Circulation. 92:3172-3177.

22. Laufs, U., and Liao, J.K. 1998. Post-transcriptional regulation of endothe- 
lial nitric oxide synthase mRNA stability by Rho GTPase. J. Biol. Chem. 273:24266-24271.

23. Kureishi, Y., et al. 2000. The HMG-CoA reductase inhibitor simvastatin activates the protein kinase Akt and promotes angiogenesis in normocholesterolemic animals. Nat. Med. 6:1004-1010.

24. Dimmeler, S., and Zeiher, A.M. 2000. Akt takes center stage in angiogenesis signaling. Circ. Res. 86:4-5.

25. Dimmeler, S., et al. 1999. Activation of nitric oxide synthase in endothelial cells via Akt-dependent phosphorylation. Nature. 399:601-605.

26. Takakura, N., et al. 2000. A role for hematopoietic stem cells in promoting angiogenesis. Cell. 102:199-209.

27. Weissman, I.L. 2000. Stem cells: units of development, units of regeneration, and units in evolution. Cell. 100:157-168.

28. Gerber, H.P., et al. 1998. Vascular endothelial growth factor regulates endothelial cell survival through the phosphatidylinositol 3'-kinase/Akt signal transduction pathway. Requirement for Flk-1/KDR activation. $J$. Biol. Chem. 273:30336-30343.

29. Laufs, U., and Liao, J.K. 2000. Targeting rho in cardiovascular disease. Circ. Res. 87:526-528.
30. Baller, D., et al. 1999. Improvement in coronary flow reserve determined by positron emission tomography after 6 months of cholesterol-lowering therapy in patients with early stages of coronary atherosclerosis. Circulation. 99:2871-2875.

31. Schwartz, G.G., et al. 2001. Effects of atorvastatin on early recurrent ischemic events in acute coronary syndromes: the MIRACL study. A randomized controlled trial. JAMA. 285:1711-1718.

32. Dimmeler, S., Assmus, B., Hermann, C., Haendeler, J., and Zeiher, A.M. 1998. Fluid shear stress stimulates phosphorylation of Akt in human endothelial cells: involvement in suppression of apoptosis. Circ. Res. 83:334-342.

33. Fulton, D., et al. 1999. Regulation of endothelium-derived nitric oxide production by the protein kinase Akt. Nature. 399:597-601.

34. Dimmeler, S., Dernbach, E., and Zeiher, A.M. 2000. Phosphorylation of the endothelial nitric oxide synthase at Ser 1177 is required for VEGFinduced endothelial cell migration. FEBS Lett. 477:258-262.

35. Morales-Ruiz, M., et al. 2000. Vascular endothelial growth factor-stimulated actin reorganization and migration of endothelial cells is regulated via the serine/threonine kinase Akt. Circ. Res. 86:892-896. 